Research Article

\title{
The Therapeutic Effect of Electroacupuncture Therapy for Ischemic Stroke
}

\author{
Bao-Hai Yu, ${ }^{1}$ Ying Xing, ${ }^{2}$ and Feng Zhang $\mathbb{D}^{2,3}$ \\ ${ }^{1}$ Department of Radiology, The Third Hospital of Hebei Medical University, Shijiazhuang 050051, China \\ ${ }^{2}$ Department of Rehabilitation Medicine, The Third Hospital of Hebei Medical University, Shijiazhuang 050051, China \\ ${ }^{3}$ Hebei Provincial Orthopedic Biomechanics Key Laboratory, The Third Hospital of Hebei Medical University, \\ Shijiazhuang 050051, China \\ Correspondence should be addressed to Feng Zhang; ydsyzf@hebmu.edu.cn
}

Received 5 July 2020; Revised 9 September 2020; Accepted 18 September 2020; Published 29 September 2020

Academic Editor: Mark Moss

Copyright (c) 2020 Bao-Hai Yu et al. This is an open access article distributed under the Creative Commons Attribution License, which permits unrestricted use, distribution, and reproduction in any medium, provided the original work is properly cited.

\begin{abstract}
Electroacupuncture (EA) stimulation is a supplementary therapy and commonly applied in treatment of ischemic stroke in clinic. Stroke is an important cause of long-term disability in individuals in both developing and developed countries. In our review, we show the application of EA stimulation for apoplectic pain, limbs spasticity, blood flow interruption, depression, swallowing dysfunction, aphasia, urinary incontinence, cognition and memory impairment, and constipation following stroke in patients and the related mechanisms in animals. The effectiveness of EA involves with acupoints, intensity, intervals, and duration of intervention for treatment of stroke. The combination of EA and common rehabilitation treatment may exert better effect compared with EA alone. In summary, EA might provide a potential treatment strategy for treating apoplectic patients in clinic.
\end{abstract}

\section{Introduction}

Ischemic stroke is a usual cerebrovascular illness and a leading cause of disabilities and death worldwide, accounting for approximately $87 \%$ of all stroke patients. Also known as brain attack, patients may suddenly suffer from incoherent speech, paralysis, or loss of vision owing to interrupting blood flow (ischemia) resulted from embolism or thrombosis [1]. Ischemic stroke induces a decrease in cerebral blood flow, which is enough to impair normal cellular function $[2,3]$. Fast reperfusion is a crucial therapy method for patients with acute ischemic stroke but usually results in cerebral ischemia/reperfusion injury [4]. Therefore, a feasible therapeutic method that attenuates the poststroke neural deficits still is essential in the clinical setting. Acupuncture has been used in treating cerebral diseases and mental disorders for a long time $[5,6]$. Electroacupuncture (EA) is another type of acupuncture, originating from the combination of acupuncture and electrical stimulation. As a relatively feasible, simple, and cheap therapy, it is commonly accepted by stroke patients in clinic
[7]. The clinical effectiveness of EA in stroke rehabilitation has been demonstrated in numerous studies [8-10].

\section{The Therapeutic Effect of EA for Ischemic Stroke in Experimental Animals}

2.1. The Effect of EA on Neurocytes in Animals. EA may prompt neuronal regeneration, migration of newborn neuron, and their maturation in the ischemic brain striatum of rats [11]. EA stimulation at Dazhui (GV 14) and Baihui (GV 20) four-day postischemia (subacute stage) can enhance astrogliosis and neurogenesis, which likely contributed to long-term functional recovery after focal cerebral ischemia [12]. Deng et al. show that EA stimulation may increase new projections and axon regeneration from the corticospinal tract at $28 \mathrm{~d}$ following ischemic stroke in rats [13]. The vagus dorsal motor nucleus, as the largest origin of parasympathetic preganglionic neurons, may be activated by EA in the lower brainstem, and parasympathetic dysfunction may inhibit these abovementioned alterations, indicating that EA may be an alternative therapy to activate the 
parasympathetic nervous system after stroke [14]. Han et al. indicate that EA may be involved in activation of astrocytes in peri-ischemic brain, promotion of the recovery of behavioral deficits, and prevention of excess reactive gliosis after ischemic stroke [15]. EA stimulation at Renzhong may exert benefits in improvement of motor function and the motor cortical excitability following ischemic stroke [16]. Si et al. show that EA may prompt somatosensory evoked potential of rats following ischemic stroke [17]. EA treatment at points of Quchi and Zusanli can increase the functional connectivity between the ipsilateral motor cortex and the motor function-related brain regions, consisting of the motor cortex, striatum, and sensory cortex in focal ischemic rats [18].

2.2. The Effect of EA on Cerebral Angiogenesis and Blood Flow in Animals. Du et al. suggest that EA might play a crucial role on promotion of angiogenesis in cerebral ischemic rats [19]. Shi et al. also show that EA at Shuigou (GV26) enhances angiogenesis and establishment of collateral circulation and prompts neurological function [20]. Increased expression of apelin-APJ protein and mRNA induced by EA $(15 \mathrm{~Hz}, 2 \mathrm{~mA})$ applied at Shuigou (GV 26) exerted a crucial role in cerebral ischemic rats which maybe involved in facilitated collateral circulation and blood vessel regeneration [21]. EA at Yin meridian acupoints can significantly facilitate neurobehavioural functional recovery, which is associated with increased vascular density and enhanced vascular endothelial growth factor (VEGF) expression and protein kinase B/endothelial nitric oxide synthase (Akt/ eNOS) phosphorylation in the peri-ischemia cortex of rats [22]. Liu et al. show that EA can balance miRNA levels, such as mir-328 and mir-126, so as to promote angiogenesis in ischemic cortex via regulating expression of VEGF family genes and proteins [23]. Furthermore, Hsieh et al. show that EA with a frequency of 2 and $15 \mathrm{~Hz}$ at Zusanli acupoints in both two legs may lead to the enhancement of cerebral blood flow in normal or ischemic stroke rat [24]. Zhou et al. demonstrate that EA intervention may exert brain protection via rapidly upregulating blood flow of the infarction region [25].

\subsection{The Effect of EA on Improving Motor Dysfunction of} Animals. EA with low frequency at Shuigou acupoint may exert obvious effect to prompt motor functional recovery in rats following ischemic stroke [26]. Liu and Lai demonstrate that EA plays a critical role in treatment of ischemic brain injury in the early stage of stroke and may effectively alleviate ischemic pathological damage, infarct volume, and neurologic deficit [27]. Liu et al. also suggest that EA at the points of ST36 and LI11 may reduce the infarct volumes, alleviate neurological deficit, and improve motor dysfunction [28]. It should be noted that many clinical research has identified that there can be no direct evidence/relationship between the infarct volume change/difference and the functional recovery. Therefore, rationale of reduction in the infarct volume by electroacupuncture therapy is not clear.
2.4. The Effect of EA on Autophagy and Apoptosis in Animals. EA treatment at points of Quchi and Zusanli can exert protective effects in rats with cerebral ischemia/reperfusion injury, associating with the inhibition of neuronal autophagy and apoptosis through activating the PI3K/AKT/mTOR pathway [29]. EA may also effectively alleviate central poststroke pain and suppress autophagy in the hippocampus through reducing $\beta$-catenin/COX-2 protein levels [30]. Xing et al. show that the neuroprotective effect induced by EA treatment against cell apoptosis in ischemic brain might associated with upregulation of midkine and regulation of ERK/JNK/p38 signal pathway [31].

\subsection{The Effect of EA on Cerebral Edema and Blood-Brain} Barrier (BBB) in Animals. Jung et al. show that EA pretreatment alleviates cerebral edema and blood-brain barrier (BBB) destruction, which may improve neural function. The $\mathrm{BBB}$ recovery by EA pretreatment might be associated with reduction of NOX4 expression and ROS generation [32]. Zhang et al. show that EA may improve brain edema in rats with ischemic stroke [33]. The inhibition of cerebral edema and $\mathrm{BBB}$ permeability induced by EA pretreatment was correlated with inhibition of $p$-caveolin-1 expression and alleviation of tight junction protein degradation and in the endothelial cells [34].

2.6. The Effect of EA on Other Aspects in Animals. Acupuncture treatment is a crucial part of Chinese traditional medicine and its feasible analgesic effect is widely accepted worldwide [35]. Lin et al. show that EA at Shenting and Baihui acupoints exerts a beneficial effect in promoting the cognitive function recovery after cerebral ischemic stroke [36]. EA may decrease the episodes of spreading depression after cerebral ischemic stroke, which may involve in the reduction of infarct volume of ischemic brain [36]. EA at Shuigou (GV26) significantly improved the neurological deficit symptoms in rats with ischemic stroke, which may be involved in upregulating Wnt7a and LEF1 proteins and mRNAs levels and decreasing GSK- $3 \beta$ and DKK1 proteins and mRNAs levels [37]. Jiang et al. showed a novel antiinflammatory mechanism induced by EA via $\alpha 7 \mathrm{nAChR}$ mediated inhibition of NLRP3 inflammasome in rats after cerebral ischemic injury [38].

\section{The Therapeutic Effect of EA for Ischemic Stroke in Clinic}

3.1. The Effect of EA on Central Nervous System in Stroke Patients. EA at head acupoints in stroke patients may contribute to the stimulation of nerve tissue involved with motion via activating the bilateral cerebral motor areas. Furthermore, in six right-handed stroke patients, EA stimulation at Baihui (GV 20) and right Qubin for twenty minutes may also activate other neural regions, suggesting that injured motor functional reorganization is a neural network behavior, and EA may affect several aspects of neural network so as to further promote motor function recovery [39]. Both exercise and EA may promptly improve 
somatosensory evoked potential of stroke patients in the recovery stage, and the Bobath therapy in combination with EA stimulation was proved to improve cerebral function in stroke patients [40]. Si et al. suggest that EA may improve the neurological function in patients with acute ischemic stroke [17]. Ho et al. demonstrate that EA exerts beneficial effects in stroke, and it might be a suitable nondrug therapy for mobilization of stem cells in CNS [41]. Ouyang et al. suggest that EA of $2 / 15 \mathrm{~Hz}$ and $100 \mathrm{~Hz}$ exerts better benefits in improving brain cell functions and local cerebral blood perfusion than that of EA of $2 \mathrm{~Hz}$ according to the results of single photon emission computed tomography (SPECT) [42].

\subsection{The Effect of EA on Poststroke Psychological Illness fol-} lowing Stroke. Man et al. suggest that the dense cranial EA intervention combined with body acupuncture with $2 \mathrm{~Hz}$ at 9 volts for 30 minutes at Baihui (GV 20), Yintang (EX-HN 3), Hegu (LI 4), and Quchi (LI 11) might be a feasible therapy for poststroke neuropsychiatric sequelae [43]. Wu and Liu demonstrate that acupuncture at Taichong (LR 3), Shenting (GV 24), GV20, EX-HN 3, GV26, and LI 4, as an effective and crucial therapy, may effectively improve the symptom of poststroke anxiety neurosis (PSAN). The total effective rate of acupuncture stimulation was $82.35 \%$ [44]. Tang et al. suggest that the low-frequency EA treatment at the aupoints of Dazhui (GV 14) and Shenshu (BL 23) exerts similar effect for poststroke insomnia to oral medication of estazolam as a secure and effective therapy [45].

Poststroke depression (PSD) is characterized by anxiety, disordered sleep, hopelessness, and lowered responsiveness and is a common stroke complication [46]. Cai et al. show that EA stimulation might be safe and effective for treating poststroke depression (PSD) in clinic [8]. Acupuncture plus auricular point sticking are effective and safe for poststroke depression (PSD). During course of treatment, acupuncture was applied at Baihui (GV 20), Shenting (GV 24), Sishencong (EX-HN 1), Yintang (GV 29), Neiguan (PC 6), Shenmen (HT 7), Taichong (LR 3), Zusanli (ST 36), Hegu (LI 4), Fenglong (ST 40), and Sanyinjiao (SP 6). This combination treatment may improve the clinical symptoms as evidenced by lowered scores, including the sleep disturbance factor, anxiety/somatization factor, and hopelessness factor [47]. EA plus body acupuncture has positive effect on alleviating PSD and cognitive deterioration after stroke, particularly with electrical stimulation on forehead acupoints [48]. Wrist-ankle acupuncture plus fluoxetine can mitigate the depression symptoms after stroke. Moreover, wrist-ankle acupuncture stimulation can increase the antidepressant effect of fluoxetine [49].

\subsection{The Effect of EA on Improving Spasticity following Stroke.} Spasm is the commonest poststroke complication, and its occurrence rate is 20-40 percent in stroke survivors [50]. Moon et al. demonstrate that EA at Shousanli (LI 10), Waiguan (TE 5), LI 11, and LI 4 may transiently alleviate spasticity following stroke, and repeated EA stimulation may sustain the effect of mediating spasticity [51]. Wu indicates that EA treatment at the nerve trunk may significantly facilitate the limbs functional recovery and reduce the rate of disability at the spastic phase of poststroke hemiplegic patients [52]. In addition, the combined application of EA and acupuncture produced a better effect in improving hand spasm, alleviating hand dysfunction and upregulating the quality of life for patients with stroke compared with simple acupuncture [10]. Furthermore, the combination of EA and rehabilitation therapy plays a critical role in regulating lower limbs spasticity in poststroke patients [53]. Wang et al. show that 6-week EA at Zeqian (EX-UE, A32), Shounizhu (EXUE), Shaohai (HT3), and Neiguan (PC6) in affected side, combined with standard rehabilitation treatment, may decrease the elbow spasticity of chronic stroke survivors [54]. EA at LI 4, Houxi (SI 3), TE 5, LI 11, LI 10, and Jianyu (LI 15), in combination with muscle strengthening training for 6 weeks, may obviously alleviate spasticity of the wrist joint in chronic stroke patients [55]. Liu et al. show that EA plus strength training may promote motor function recovery and alleviate muscle spasticity for moderate or severe muscle spasticity in chronic stroke patients [56].

\subsection{The Effect of EA on Improving Limbs Function following} Stroke. According to enhancement of the upper limbs function, traditional Chinese acupuncture may be beneficial for improving chronic stroke symptoms in patients [57]. Zhao et al. also show that Jingin acupuncture at GV 26, GV 20 , and PC 6 may effectively enhance daily-life ability via improving subtle activity of hemiplegic hand in the phase of poststroke recovery [58]. However, Yang et al. demonstrated that EA therapy may exert beneficial effect in the upperextremity function following ischemic stroke and provide a better effect than simple manual acupuncture [59]. Hsieh et al. indicate that EA at GV 20, Fengchi (GB 20), LI 15, LI 11, LI 4, Fengshi (GB 31), Yanglingquan (GB 34), and ST 36 on the affected side, may effectively promote motor function recovery, particularly in upper extremity motor function and in patients with the primary ischemic stroke [60]. EA at LI 15, LI 4, TE 5, and LI 10, combined with exercise training, may improve arms and legs function in poststroke hemiplegia patients [61]. Moreover, Liu and Xiao show that EA at Juci and Tanci may improve nail-bed microcirculation in hemiplegic side of poststroke patients, and the effect of Juci stimulation is better Tanci stimulation [62]. Wang suggests that EA at acupoints of different channels exerts benefits on poststroke hemiplegia patients at different stages of stroke [63].

Chen et al. suggest that EA as a supplemental therapy may exert benefits in apoplexy patients with shoulder subluxation [64]. EA treatment at Jianwaishu (SI 14), Jianzhen (SI 9), Naoshu (SI 10), Binao (LI 14), and Bingfeng (SI 12) with intermittent wave and common rehabilitation therapy exerts a better effect compared to continuous and disperse-dense wave for the treatment of shoulder subluxation, and the combination treatment may effectively prompt shoulder functional recovery and improve subluxation [65]. The combination of EA at LI 15, Jianliao (TE 14), and SI 9 and rehabilitation techniques also may exert 
benefits in regulating the muscular tension of shoulder joint and the muscles around the scapula and muscle strength and improving the shoulder subluxation [66].

In addition, acupuncture intervention at lateral side of BL 10 associated with scalp points, including Zhenxiapangxian (MS 14) and Dingnieqianxiexian (MS 6), plays a critical role in walking ability and standing balance ability after stroke [67]. EA at bilateral MS 6 plays important role in recovery of nerve defects in the hemiplegic patients following acute ischemic brain injury, enhancing limb motor function and the daily-life activity ability [68]. EA $(20 \mathrm{~Hz}$, $2 \mathrm{~mA}$ ) at GV 20, EX-HN 3, GV 26, LI 4, ST 36, SP 6, and Taichong(LR 3), with cupping at the lumboback, exerts a better effect than medication in relief of fatigue in poststroke patients [69]. Liu et al. indicated that EA at Pishu (BL 20), Shenshu (BL 23), Dachangshu (BL 25), and Qihaishu (BL 24) may elevate the single-foot supporting phase rate in stroke patients [70].

3.5. The Effect of EA on Improving Swallowing after Stroke. EA stimulation as a feasible and effective therapy may alleviate swallowing dysfunction following stroke at Chonggu acupoints with deep insertion [71]. EA treatment integrated with swallowing functional training may promote the recovery of swallowing ability in poststroke patients with dysphagia [9]. However, Huang et al. show that either electric stimulation or acupuncture at GB 20, LI 18, threeneedles on the forehead, etc. combined with rehabilitation training exerts a better effect compared with simple rehabilitation training. The effect of acupuncture in dysphagia is equal to that of electric stimulation [72]. EA at eight-neckocciput acupoints exerts a better effect on improving swallowing of medulla oblongata palsy following brainstem infarction compared with the routine acupoints [73]. In addition, Su et al. show that EA at Yamen (GV 15), bilateral GB 20, bilateral Renying (ST 9), bilateral Sanyinjiao (SP 6), bilateral LI 4, and bilateral Fenglong (ST 40), may effectively enhance the spleen, clear phlegm, dredge the channels, clean dampness, bring out resuscitation, increase cerebral blood flow, alleviate brain edema, reduce cerebrovascular spasm, promote anoxic tolerance of neuronal cells, and regulate internal organs functions in patients with poststroke dysphagia [74].

3.6. The Effect of EA on Speech Apraxia after Stroke. Speech rehabilitation training associated with the scalp electric acupuncture $(2 \mathrm{~mA}, 50 \mathrm{~Hz})$ in Broca's area under anatomic orientation for four weeks may obviously relieve the speech disorder in with poststroke speech apraxia patients (18 cases with cerebral hemorrhage (lesion of 15 cases in the left basal ganglia, lesion of 1 case in the left frontal temporal and parietal, lesion of 2 cases in the left side of the basal ganglia and thalamus) and 42 cases with cerebral infarction (lesion of 11 cases in left bottom of the base section, 12 cases in left frontotemporal top, 11 cases in left insula temporal lobe, 5 cases in left insula and left ventricle narrator, and 3 cases in left frontal lobe and insular lobe) [75]. Chang et al. demonstrate that the stimulation of
Xuanzhong and Tongli acupoints provides a therapeutic effect on the aphasia recovery after stroke via activating several brain regions related to language in poststroke aphasic patients [76]. In comparison with the routine acupoints, EA at eight-neck-occiput points plays a better role on speech disability of medulla oblongata palsy following brainstem infarction [73].

\subsection{The Effect of EA on Improving Cognition and Memory} after Stroke. Chou et al. show that EA at PC6 and Shenmen (HT7) for twenty minutes twice a week for eight weeks may improve the recovery of cognition function and life quality in poststroke patients [77]. Based on the rehabilitation training and conventional medication, EA stimulation at Dingniehouxiexian (MS 7), bilateral Ezhongxian (MS 1), Xuanzhong (GB 39), Dingzhongxian (MS 5), LI 4, Taichong (LR 3), ST 36, Taixi (KI 3), and GB 20 five times per week for eight weeks may promote recovery of memory function and the metabolism of cerebral tissue in the poststroke patients (infarct regions: 19 cases of basal ganglia, 9 cases of lateral ventricle, 1 case of thalamus, and 1 case of brainstem), and it has a better effect compared to medication associated with rehabilitation training [78]. Zeng et al. also show that acupuncture at GV 20, EX-HN 1, GV 24, GV 29, LI 4, LR 3, EX-HN 1, GV 24, and GV 29, five times per week for eight weeks can prompt the recovery of cognitive function and improve daily-life ability in subacute stroke patients with mild cognitive dysfunction on the basis of the traditional therapy and the cognitive rehabilitation training [79].

3.8. The Effect of EA on Easing Pain after Stroke. EA exerts effective benefits in well-being and pain control via activating antinociceptive pathway in the brain of patients with a history of ischemia in the left temporoparietal region [80]. EA at LI15 and LI 4, plus either penetration needling or routine acupuncture, may exert benefits in improvement of motion function and alleviation of edema and pain for patients with poststroke shoulder-hand syndrome [81]. Chau et al. show that EA treatment may be effective for patients with poststroke shoulder pain to ease the pain, promote upper limbs function, and improve physical function [82]. EA stimulation at Huatuojiaji points may obviously improve postapoplectic thalamic spontaneous pain [83]. Li et al. indicate that EA at Chize (LU 5), LI 15, TE 14, Quze (PC 3), Jianjing (GB 21), and Shaohai (HT 3), in association with Tuina exerts a better effect on poststroke shoulder pain than comprehensive rehabilitation treatment such as the electrostimulation in patients [84].

3.9. The Effect of EA on Improving Urinary Function after Stroke. In comparison with indwelling catheter therapy, EA stimulation at Qugu (CV 2), Zhongji (CV 3), Shuidao (ST 28), Qihai (CV 6), and Guanyuan (CV 4) has a better effect in promoting bladder capacity and attenuating apoplectic urinary incontinence in poststroke patients with urinary incontinence [85]. EA treatment (1 Hz, $15 \mathrm{~min})$ at Sanyinjiao (SP6), Ciliao (BL32), and Pangguangshu (BL28) might be a 


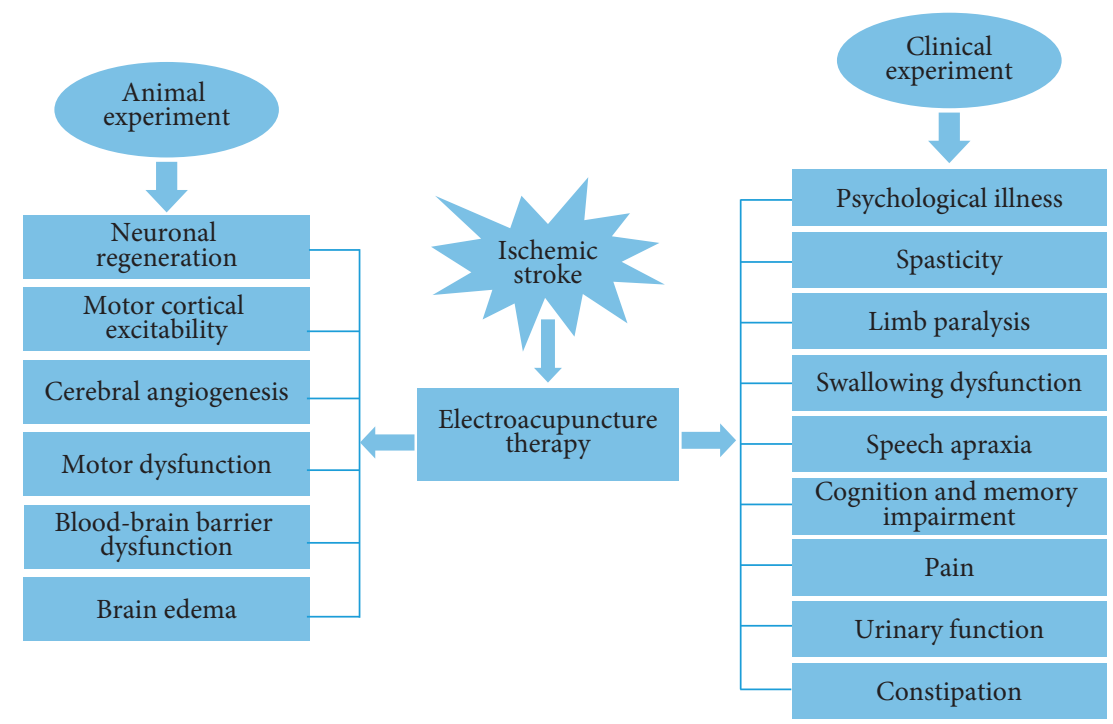

FIGURE 1: Electroacupuncture could promote neuronal regeneration, increase motor cortical excitability, improve cerebral angiogenesis, reduce motor dysfunction, decrease brain edema, and alleviate the impairment of blood-brain barrier according to the results of animal experiments. Meanwhile, electroacupuncture could improve a series of dysfunctions following stroke, including constipation, urinary function, pain, cognition and memory impairment, speech apraxia, swallowing disability, limb paralysis, spasticity, and psychological illness according to the results of clinical trial.

safe therapy for improvement of urinary function because of the effective effects induced by EA on stroke patients with incomplete bladder emptying [86]. EA at Jianyu (LI 15), Xuehai (SP 10), Shenshu (BL 23), Huiyang (BL 35) may also improve micturition clinical symptom and attenuate urinary incontinence severity in stroke patients [87]. Liu et al. indicate that EA intervention at Huiyang and Baliao provides a beneficial effect in alleviating detrusor overactivity after stroke by markedly mitigating symptoms of lower urinary tract, improving bladder compliance and cystometric capacity, reducing upper urinary tract injury risk, and alleviating pressure of detrusor leak point [88].

\subsection{The Effect of EA on Improving Constipation after Stroke.} Abdominal EA treatment at Daheng (SP 15), Fujie (SP 14), Tianshu (ST 25), Shuidao (ST 28), etc. may effectively improve poststroke constipation and accelerate gastrointestinal movement in patients with stroke [89]. Wang et al. show that basic comprehensive treatment in combination with EA at the point of Zusanli (ST 36) and Tian-shui (ST 25) plays a key role in prevention and treatment of constipation symptom in the acute phase of ischemic stroke [90].

3.11. The Effect of EA on Other Aspects after Stroke. Fu et al. show that EA at Jianyu (LI 15), Biguan (ST 31), Hegu (LI 4), Taichong (LR 3), Quchi (LI 11), Yanglingquan (GB 34), and Shenshu (BL 23), combined with dissolve-stasis herbs, rehabilitation training, and active-blood herbs, may be effective for treating ischemic stroke in clinic [91]. Electrospoon needles or electrofiliform needle may effectively promote motor dysfunction and daily-life ability in ischemic stroke patients [92]. Qian et al. show that acupuncture intervention at Jiquan (HT 1), Quchi (LI 11), Hegu (LI 4), Huantiao (GB 30), etc., twice per day in convalescence of cerebral infarction may exert more benefits than once per day in patients [93]. Li shows that EA at acupoints of either Yin Meridians or Yang Meridians may induce protection in poststroke patients [94]. Wong et al. suggest that EA via adhesive surface electrodes combined with appropriate rehabilitation therapy is an effective and convenient treatment for stroke patients [95]. EA and acupoint injection may significantly elevate daily-life ability and improve the neural function for the ischemic stroke patients, exerting a better effect than that EA alone [96]. Li et al. indicate that the combination therapy of EA at GV 20, Shenzhu (GV 12), Tianding (LI 17), LI 10, Biguan (ST 31), and Fenglong (ST40) and intracarotid drug injection may increase the cerebral blood vessels elasticity, promote vasodilation, and elevate the cerebral blood flow, contributing to sufficient supply of blood and oxygen and recovery of ischemic brain function following cerebral infarction [97].

Pei et al show that EA stimulation plays an important role in improving life quality and in health care, social services, and daily living ability of patients in acute stage EA stimulation at LI 4, LI 10, LI11, LI15, SP 6, Fenglong (ST 40), ST 36, and DU20 may prompt motor function recovery and then improve the living activities in the early stage of stroke [98]. Wang et al. suggest that EA treatment is important in improving the nervous dysfunction deficits following fourweek intervention and enhancing the daily-life activity level following six-month follow-up visit, and systematic acupuncture treatment may alleviate the occurrence rate of secondary apoplexy in patients [99].

\section{Conclusion}

In summary, as demonstrated in Figure 1, EA treatment or preconditioning may play an important role in alleviating edema, easing pain, enhancing cerebral blood flow and 
daily-life ability, improving cognition and memory function, speech function, swallowing function, motor function, as well as nerve, intestinal, and urinary system. In addition, EA stimulation combined with other common rehabilitation treatment might exert better effect for treatment of stroke than EA alone. EA with high frequency or long duration may elicit effective improvement in apoplectic patients. The effect of EA stimulation also involves acupoints, intensity, and interval of stimulation. All of those mentioned above provide a potential treatment strategy for treating apoplectic patients in clinic.

\section{Data Availability}

This is a review article with no underlying data.

\section{Conflicts of Interest}

The authors declare that there are no conflicts of interest.

\section{References}

[1] M. A. Moskowitz, E. H. Lo, and C. Iadecola, "The science of stroke: mechanisms in search of treatments," Neuron, vol. 67, no. 2, pp. 181-198, 2010.

[2] M. Wang, Y.-J. Li, Y. Ding et al., "Silibinin prevents autophagic cell death upon oxidative stress in cortical neurons and cerebral ischemia-reperfusion injury," Molecular Neurobiology, vol. 53, no. 2, pp. 932-943, 2016.

[3] P. Wicha, J. Tocharus, A. Janyou et al., "Hexahydrocurcumin protects against cerebral ischemia/reperfusion injury, attenuates inflammation, and improves antioxidant defenses in a rat stroke model," PLoS One, vol. 12, no. 12, Article ID e0189211, 2017.

[4] J. F. Wang, Z. G. Mei, Y. Fu et al., "Puerarin protects rat brain against ischemia/reperfusion injury by suppressing autophagy via the AMPK-mTOR-ULK1 signaling pathway," Neural Regeneration Research, vol. 13, no. 6, pp. 989-998, 2018.

[5] G. X. Ni and Y. Y. Song, "Exploration of xingnao tiaochang acupuncture therapy in treatment of Parkinson's disease on the base of brain-gut axis theory," Zhongguo Zhen Jiu, vol. 40, no. 3, pp. 315-317, 2020.

[6] B. R. Stacey, R. E. Williams, R. G. Buckner, and J. A. Hair, "Changes in weight and blood composition of hereford and brahman steers in drylot and infested with adult gulf coast ticks12," Journal of Economic Entomology, vol. 71, no. 6, pp. 967-970, 1978.

[7] J. Zhan, R. Pan, M. Zhou et al., "Electroacupuncture as an adjunctive therapy for motor dysfunction in acute stroke survivors: a systematic review and meta-analyses," BMJ Open, vol. 8, no. 1, Article ID e017153, 2018.

[8] W. Cai, W. Ma, G. T. Wang, and W. D. Shen, "Efficacy and safety of electroacupuncture for post stroke depression: study protocol for a randomized controlled trial," Trials, vol. 19, no. 1, p. 152, 2018.

[9] S. Y. Zhang, S. B. Liu, W. Wu et al., "Clinical trials for treatment of stroke patients with dysphagia by vitalstim electroacupuncture combined with swallowing rehabilitation training," Zhen Ci Yan Jiu, vol. 42, no. 2, pp. 168-172, 2017.

[10] M. Tian, T. Lou, and J. Leng, "Clinical observation of acupuncture plus electroacupuncture for hand spasm in stroke patients," Zhongguo Zhen Jiu, vol. 37, no. 9, pp. 932-935, 2017.
[11] Z. J. Yang, D. H. Shen, X. Guo, and F. Y. Sun, "Electroacupuncture enhances striatal neurogenesis in adult rat brains after a transient cerebral middle artery occlusion," $A c u$ puncture \& Electro-Therapeutics Research, vol. 30, no. 3-4, pp. 185-199, 2005.

[12] P. Young-Wook, H. Gi Yoon, K. Min Jae, L. Seo-Yeon, C. Byung Tae, and S. Hwa Kyoung, "Subacute electroacupuncture at baihui (GV 20) and dazhui (GV 14) promotes post-stroke functional recovery via neurogenesis and astrogliosis in a photothrombotic stroke mouse model," Journal of Traditional Chinese Medicine, vol. 39, no. 6, pp. 833-841, 2019.

[13] B. Deng, F. Bai, H. Zhou et al., "Electroacupuncture enhances rehabilitation through miR-181b targeting PirB after ischemic stroke," Scientific Reports, vol. 6, p. 38997, 2016.

[14] L. Chi, K. Du, D. Liu, Y. Bo, and W. Li, "Electroacupuncture brain protection during ischemic stroke: a role for the parasympathetic nervous system," Journal of Cerebral Blood Flow \& Metabolism, vol. 38, no. 3, pp. 479-491, 2018.

[15] X. Han, X. Huang, Y. Wang, and H. Chen, "A study of astrocyte activation in the periinfarct region after cerebral ischemia with electroacupuncture," Brain Injury, vol. 24, no. 5, pp. 773-779, 2010.

[16] H. Lin, W. Shu, M. Jinquan, Y. Wenping, S. Yan, and S. Xuemin, "The effects of electro-acupuncturing DU26 (renzhong) on motor cortical excitability and neurofunction after focal cerebral ischemia injury in rats," Acupuncture \& Electro-Therapeutics Research, vol. 35, no. 1-2, pp. 29-44, 2010.

[17] Q.-M. Si, G.-C. Wu, and X.-D. Cao, "Effects of electroacupuncture on acute cerebral infarction," Acupuncture \& Electro-Therapeutics Research, vol. 23, no. 2, pp. 117-124, 1998.

[18] Z. Li, M. Yang, Y. Lin et al., "Electroacupuncture promotes motor function and functional connectivity in rats with ischemic stroke: an animal resting-state functional magnetic resonance imaging study," Acupuncture in Medicine, Article ID 964528420920297, 2020.

[19] Y. Du, L. Shi, J. Li, J. Xiong, B. Li, and X. Fan, "Angiogenesis and improved cerebral blood flow in the ischemic boundary area were detected after electroacupuncture treatment to rats with ischemic stroke," Neurological Research, vol. 33, no. 1, pp. 101-107, 2011.

[20] L. Shi, H. M. Cao, Y. Li et al., "Electroacupuncture improves neurovascular unit reconstruction by promoting collateral circulation and angiogenesis," Neural Regeneration Research, vol. 12, no. 12, pp. 2000-2006, 2017.

[21] L. H. Yang, Y. H. Du, and J. Li, "Effect of electroacupuncture on expression of apelin-APJ system of cerebral vascular endothelial cell in rats with cerebral infarction," Zhen Ci Yan Jiu, vol. 42, no. 1, pp. 9-13, 2017.

[22] L. Liu, Q. Zhang, H.-Y. Xie et al., "Differences in post-ischemic motor recovery and angiogenesis of MCAO rats following electroacupuncture at different acupoints," Current Neurovascular Research, vol. 17, no. 1, pp. 71-78, 2020.

[23] L. Liu, N. H. Wang, Q. Zhang, S. Y. Li, W. J. Gu, and Y. Wu, "Micro-ribonucleic acids participate in electroacupuncture intervention-induced improvement of ischemic stroke," Zhen Ci Yan Jiu, vol. 44, no. 9, pp. 686-692, 2019.

[24] C.-L. Hsieh, Q.-Y. Chang, I.-H. Lin et al., "The study of electroacupuncture on cerebral blood flow in rats with and without cerebral ischemia," The American Journal of Chinese Medicine, vol. 34, no. 02, pp. 351-361, 2006.

[25] F. Zhou, J. Guo, J. Cheng, G. Wu, and Y. Xia, "Electroacupuncture increased cerebral blood flow and reduced 
ischemic brain injury: dependence on stimulation intensity and frequency," Journal of Applied Physiology, vol. 111, no. 6, pp. 1877-1887, 2011.

[26] W.-P. Yao, S. Wang, L. Han, J.-Q. Ma, and Y. Shen, "Effects of different frequencies of electro-acupuncture at shuigou (GV 26) on recovery of motor function in rats with focal cerebral ischemic injury," Journal of Traditional Chinese Medicine, vol. 32, no. 1, pp. 99-104, 2012.

[27] Z. Liu and X. S. Lai, "Effects of electroacupuncture on neurological deficit and pathomorphologic lesion in the rat of focal cerebral ischemia," Zhongguo Zhen Jiu, vol. 25, no. 12, pp. 879-884, 2005.

[28] W. Liu, G. Shang, S. Yang et al., "Electroacupuncture protects against ischemic stroke by reducing autophagosome formation and inhibiting autophagy through the mTORC1-ULK1 complex-beclin1 pathway," International Journal of Molecular Medicine, vol. 37, no. 2, pp. 309-318, 2016.

[29] M. M. Wang, M. Zhang, Y. S. Feng et al., "Electroacupuncture inhibits neuronal autophagy and apoptosis via the PI3K/AKT pathway following ischemic stroke," Front Cell Neurosci, vol. 14, p. 134, 2020.

[30] L. Zheng, X.-Y. Li, F.-Z. Huang et al., "Effect of electroacupuncture on relieving central post-stroke pain by inhibiting autophagy in the hippocampus," Brain Research, vol. 1733, Article ID 146680, 2020.

[31] Y. Xing, S.-D. Yang, M.-M. Wang, F. Dong, Y.-S. Feng, and F. Zhang, "Electroacupuncture alleviated neuronal apoptosis following ischemic stroke in rats via midkine and ERK/JNK/ p38 signaling pathway," Journal of Molecular Neuroscience, vol. 66, no. 1, pp. 26-36, 2018.

[32] Y. S. Jung, S. W. Lee, J. H. Park, H. B. Seo, B. T. Choi, and H. K. Shin, "Electroacupuncture preconditioning reduces ROS generation with NOX4 down-regulation and ameliorates blood-brain barrier disruption after ischemic stroke," Journal of Biomedical Science, vol. 23, p. 32, 2016.

[33] F. Zhang, Y. Wu, and J. Jia, "Electro-acupuncture can alleviate the cerebral oedema of rat after ischemia," Brain Injury, vol. 25, no. 9, pp. 895-900, 2011.

[34] R. Zou, Z. Wu, and S. Cui, "Electroacupuncture pretreatment attenuates blood-brain barrier disruption following cerebral ischemia/reperfusion," Molecular Medicine Reports, vol. 12, no. 2, pp. 2027-2034, 2015.

[35] G. H. Tian, S. S. Tao, M. T. Chen et al., "Electroacupuncture treatment alleviates central poststroke pain by inhibiting brain neuronal apoptosis and aberrant astrocyte activation," Neural Plasticity, vol. 2016, no. 1, 14 pages, Article ID 1437148, 2016.

[36] R. Lin, Y. Wu, J. Tao et al., "Electroacupuncture improves cognitive function through rho GTPases and enhances dendritic spine plasticity in rats with cerebral ischemiareperfusion," Molecular Medicine Reports, vol. 13, no. 3, pp. 2655-2660, 2016.

[37] J. J. Zhang, Y. H. Du, J. Li, L. H. Yang, L. L. Chen, and Q. P. Zha, "Effect of electroacupuncture on neurological function and Wnt signaling pathway in ischemic brain tissue of cerebral infarction rats," Zhen Ci Yan Jiu, vol. 45, no. 3, pp. 202-208, 2020.

[38] T. Jiang, M. Wu, Z. Zhang et al., "Electroacupuncture attenuated cerebral ischemic injury and neuroinflammation through alpha7nAChR-mediated inhibition of NLRP3 inflammasome in stroke rats," Molecular Medicine, vol. 25, no. 1, p. 22, 2019.

[39] Z. Fang, J. Ning, C. Xiong, and Y. Shulin, "Effects of electroacupuncture at head points on the function of cerebral motor areas in stroke patients: a pet study," Evidence-Based
Complementary and Alternative Medicine, vol. 2012, Article ID 902413, , 2012.

[40] D. E. Huang, Q. Wu, Z. R. Lin, D. Lin, F. F. Shen, and J. Z. Liu, "Effects of different interference orders of acupuncture and exercise therapy on the amplitude of somatosensory evoked potential (SEP) in the patient of hemiplegia after stroke," Zhongguo Zhen Jiu, vol. 26, no. 12, pp. 869-872, 2006.

[41] T. J. Ho, T. M. Chan, L. I. Ho et al., "The possible role of stem cells in acupuncture treatment for neurodegenerative diseases: a literature review of basic studies," Cell Transplantation, vol. 23, no. 4-5, pp. 559-566, 2014.

[42] G. Ouyang, S. W. Jia, F. Wang, Y. Shi, and Z. Gao, "Effects of electroacupuncture of different frequencies on cerebral blood perfusion and cerebral function in the patient of stroke," Zhongguo Zhen Jiu, vol. 25, no. 11, pp. 776-778, 2005.

[43] S. C. Man, B. H. Hung, R. M. Ng et al., "A pilot controlled trial of a combination of dense cranial electroacupuncture stimulation and body acupuncture for post-stroke depression," BMC Complementary Medicine and Therapies, vol. 14, p. 255, 2014.

[44] P. Wu and S. Liu, "Clinical observation on post-stroke anxiety neurosis treated by acupuncture," Journal of Traditional Chinese Medicine = Chung I Tsa Chih Ying Wen pan, vol. 28, no. 3, pp. 186-188, 2008.

[45] L. Tang, C. Ma, F. You, and L. Ding, "Impacts of the lowfrequency electric stimulation at the acupoints on the content of plasma 5-HT and NE in the patients with post-stroke insomnia," Zhongguo Zhen Jiu, vol. 35, no. 8, pp. 763-767, 2015.

[46] G. C. Medeiros, D. Roy, N. Kontos, and S. R. Beach, "Poststroke depression: a 2020 updated review," General Hospital Psychiatry, vol. 66, pp. 70-80, 2020.

[47] L. Zhang, Y. Zhong, S. Quan et al., "Acupuncture combined with auricular point sticking therapy for post stroke depression:a randomized controlled trial," Zhongguo Zhen Jiu, vol. 37, no. 6, pp. 581-585, 2017.

[48] Z. J. Zhang, H. Zhao, G. X. Jin et al., "Assessor-and participant-blinded, randomized controlled trial of dense cranial electroacupuncture stimulation plus body acupuncture for neuropsychiatric sequelae of stroke," Psychiatry and Clinical Neurosciences, vol. 74, no. 3, pp. 183-190, 2020.

[49] Y. You, T. Zhang, S. Shu, X. Qian, S. Zhou, and F. Yao, "Wristankle acupuncture and fluoxetine in the treatment of poststroke depression: a randomized controlled clinical trial," Journal of Traditional Chinese Medicine = Chung I Tsa Chih Ying Wen pan, vol. 40, no. 3, pp. 455-460, 2020.

[50] S. M. Lim, J. Yoo, E. Lee et al., "Acupuncture for spasticity after stroke: a systematic review and meta-analysis of randomized controlled trials," Evidence-Based Complementary and Alternative Medicine, vol. 2015, Article ID 870398, , 2015.

[51] S.-K. Moon, Y.-K. Whang, S.-U. Park et al., "Antispastic effect of electroacupuncture and moxibustion in stroke patients," The American Journal of Chinese Medicine, vol. 31, no. 03, pp. 467-474, 2003.

[52] Y. Wu, "Effects of electroacupuncture at the nerve trunk for treatment of apoplectic hemiplegia at the spastic stage," Journal of Traditional Chinese Medicine = Chung I Tsa Chih Ying Wen pan, vol. 28, no. 3, pp. 183-185, 2008.

[53] X. B. Wang, J. Chen, T. J. Li et al., "Effect of electroacupuncture in different frequencies on electromyography and ambulation in stroke patients with lower-extremity spasticity: a randomized controlled study," Zhongguo Zhen Jiu, vol. 31, no. 7, pp. 580-584, 2011. 
[54] B. H. Wang, C. L. Lin, T. M. Li, S. D. Lin, J. G. Lin, and L. W. Chou, "Selection of acupoints for managing upperextremity spasticity in chronic stroke patients," Clinical Interventions in Aging, vol. 9, pp. 147-156, 2014.

[55] M. Mukherjee, L. K. McPeak, J. B. Redford, C. Sun, and W. Liu, "The effect of electro-acupuncture on spasticity of the wrist joint in chronic stroke survivors," Archives of Physical Medicine and Rehabilitation, vol. 88, no. 2, pp. 159-166, 2007.

[56] W. Liu, M. Mukherjee, C. Sun, H. Liu, and L. K. McPeak, "Electroacupuncture may help motor recovery in chronic stroke survivors: a pilot study," The Journal of Rehabilitation Research and Development, vol. 45, no. 4, pp. 587-596, 2008.

[57] P. M. Wayne, D. E. Krebs, E. A. Macklin et al., "Acupuncture for upper-extremity rehabilitation in chronic stroke: a randomized sham-controlled study," Archives of Physical Medicine and Rehabilitation, vol. 86, no. 12, pp. 2248-2255, 2005.

[58] Y. L. Zhao, W. C. Li, J. Huang et al., "Effects of jingjin acupuncture on fine activity of hemiplegic hand in recovery period of stroke," Zhongguo Zhen Jiu, vol. 34, no. 2, pp. 120-124, 2014.

[59] J. S. Yang, X. Gao, R. Sun et al., "Effect of electroacupuncture intervention on rehabilitation of upper limb motor function in patients with ischemic stroke," Zhen Ci Yan Jiu, vol. 40, no. 6, pp. 489-492, 2015.

[60] R. Hsieh, L. Wang, and W. Lee, "Additional therapeutic effects of electroacupuncture in conjunction with conventional rehabilitation for patients with first-ever ischaemic stroke," Journal of Rehabilitation Medicine, vol. 39, no. 3, pp. 205-211, 2007.

[61] F. F. Shen, Q. Wu, Z. R. Lin et al., "Effects of different interference orders of electroacupuncture and exercise therapy on the therapeutic effect of hemiplegia after stroke," Zhongguo Zhen Jiu, vol. 28, no. 10, pp. 711-713, 2008.

[62] G. Liu and Y. Xiao, "The influence of electric needling "juci" on nail-bed microcirculation of apoplectic patients," Zhen $\mathrm{Ci}$ Yan Jiu, vol. 15, no. 1, pp. 40-43, 1990.

[63] Q. Y. Wang, "Observation on therapeutic effect of electroacupuncture at points of different channels at different stages on hemiplagia after apoplexy," Zhongguo Zhen Jiu, vol. 26, no. 1 , pp. 33-35, 2006.

[64] C. H. Chen, T. W. Chen, M. C. Weng, W. T. Wang, Y. L. Wang, and M. H. Huang, "The effect of electroacupuncture on shoulder subluxation for stroke patients," The Kaohsiung Journal of Medical Sciences, vol. 16, no. 10, pp. 525-532, 2000.

[65] B. T. Wu and Z. H. Zhao, "Efficacy control observation on electroacupuncture with different waveforms combined with rehabilitation therapy for post-stroke shoulder subluxation," Zhongguo Zhen Jiu, vol. 33, no. 10, pp. 901-904, 2013.

[66] J. Lu, L. X. Zhang, K. J. Liu et al., "Clinical observation on electroacupuncture combined with rehabilitation techniques for treatment of shoulder subluxation after stroke," Zhongguo Zhen Jiu, vol. 30, no. 1, pp. 31-34, 2010.

[67] J. M. Chu, Y. H. Bao, and M. Zhu, "Effects of acupuncture intervention combined with rehabilitation on standing-balance-walking ability in stroke patients," Zhen Ci Yan Jiu, vol. 40, no. 6, pp. 474-478, 2015.

[68] L. Tian, X. Du, J. Wang et al., "Comparative study on the effects between manual acupuncture and electroacupuncture for hemiplegia after acute ischemic stroke," Zhongguo Zhen Jiu, vol. 36, no. 11, pp. 1121-1125, 2016.

[69] Y. Zhou, G. Y. Zhou, S. K. Li, and J. H. Jin, "Clinical observation on the therapeutic effect of electroacupuncture combined with cupping on post-stroke fatigue," Zhen Ci Yan Jiu, vol. 35, no. 5, pp. 380-383, 2010.

[70] G. Liu, C. S. Lu, and L. X. Yuan, "Study on electroacupuncture improving the single-foot supporting phase rate of stroke patients," Zhongguo Zhen Jiu, vol. 30, no. 6, pp. 446-448, 2010.

[71] Z. L. Zhang, S. H. Zhao, G. H. Chen et al., "Randomized controlled study on dysphagia after stroke treated with deep insertion of chonggu (EX-HN 27) by electroacupuncture," Zhongguo Zhen Jiu, vol. 31, no. 5, pp. 385-390, 2011.

[72] Z. Huang, F. Huang, H. X. Yan et al., "Dysphagia after stroke treated with acupuncture or electric stimulation: a randomized controlled trial," Zhongguo Zhen Jiu, vol. 30, no. 12, pp. 969-973, 2010.

[73] R. P. She and C. H. Ge, "Clinical observation on medulla oblongata palsy after brainstem infarction treated with electroacupuncture at eight-neck-occiput points," Zhongguo Zhen Jiu, vol. 34, no. 6, pp. 539-542, 2014.

[74] Y. Su, P. Li, and G. Zhao, "Electroacupuncture treatment for 45 cases of postapoplectic dysphagia," Journal of Traditional Chinese Medicine = Chung I Tsa Chih Ying Wen pan, vol. 24, no. 2, pp. 129-130, 2004.

[75] Y. Jiang, Y. Yang, R. Xiang et al., "Clinical study of post-stroke speech apraxia treated with scalp electric acupuncture under anatomic orientation and rehabilitation training," Zhongguo Zhen Jiu, vol. 35, no. 7, pp. 661-664, 2015.

[76] J. Chang, H. Zhang, Z. Tan, J. Xiao, S. Li, and Y. Gao, "Effect of electroacupuncture in patients with post-stroke motor aphasia:neurolinguistic and neuroimaging characteristics," Wien Klin Wochenschr, vol. 129, no. 3-4, pp. 102-109, 2017.

[77] P. Chou, H. Chu, and J. G. Lin, "Effects of electroacupuncture treatment on impaired cognition and quality of life in Taiwanese stroke patients," Journal of Alternative and Complementary Medicine, vol. 15, no. 10, pp. 1067-1073, 2009.

[78] J. Zhang and Y. Shen, "Magnetic resonance spectroscopic study of memory impairment after cerebral infarction treated with electroacupuncture," Zhongguo Zhen Jiu, vol. 35, no. 7, pp. 657-660, 2015.

[79] Y. Zeng, Y. Bao, M. Zhu, S. Chen, and J. Fang, "Mild cognitive impairment of stroke at subacute stage treated with acupuncture: a randomized controlled trial," Zhongguo Zhen Jiu, vol. 35, no. 10, pp. 979-982, 2015.

[80] A. B. O. Santos and J. L. Gozzani, “Acupuncture as adjuvant therapy in thalamic syndrome: case report," Brazilian Journal of Anesthesiology, vol. 61, no. 1, pp. 88-94, 2011.

[81] L. W. Xue, Q. Li, and J. Q. Sun, "Clinical observation on penetration needling combined with electroacupuncture for treatment of post-stroke shoulder-hand syndrome," Zhongguo Zhen Jiu, vol. 27, no. 7, pp. 491-493, 2007.

[82] J. P. C. Chau, S. H. S. Lo, X. Yu et al., "Effects of acupuncture on the recovery outcomes of stroke survivors with shoulder pain: a systematic review," Frontiers in Neurology, vol. 9, p. 30, 2018.

[83] Z. Jiang, C. Li, and Y. Li, "Treatment of postapoplectic thalamic spontaneous pain by electroacupuncture at huatuojiaji points," Journal of Traditional Chinese Medicine, vol. 19, no. 3, pp. 195-199, 1999.

[84] N. Li, F. W. Tian, C. W. Wang et al., "Double-center randomized controlled trial on post-stroke shoulder pain treated by electroacupuncture combined with tuina," Zhongguo Zhen Jiu, vol. 32, no. 2, pp. 101-105, 2012.

[85] F. J. Song, S. H. Jiang, S. L. Zheng et al., "Electroacupuncture for post-stroke urinary incontinence: a multi-center randomized controlled study," Zhongguo Zhen Jiu, vol. 33, no. 9, pp. 769-773, 2013. 
[86] K. W. Yu, C. L. Lin, C. C. Hung et al., "Effects of electroacupuncture on recent stroke inpatients with incomplete bladder emptying: a preliminary study," Clinical Interventions in Aging, vol. 7, pp. 469-474, 2012.

[87] J. M. Chu, Y. H. Bao, C. Zou, H. L. Zhao, Y. Gong, and C. M. Wang, "Randomized controlled clinical trials for electroacupuncture treatment of urinary incontinence in stroke patients," Zhen Ci Yan Jiu, vol. 36, no. 6, pp. 428-432, 2011.

[88] Y. Liu, L. Liu, and X. Wang, "Electroacupuncture at points baliao and huiyang (BL35) for post-stroke detrusor overactivity," Neural Regeneration Research, vol. 8, no. 18, pp. 1663-1672, 2013.

[89] D. S. Wang, S. Wang, L. L. Kong, W. Y. Wang, and X. M. Cui, "Clinical observation on abdominal electroacupuncture for treatment of poststroke constipation," Zhongguo Zhen Jiu, vol. 28, no. 1, pp. 7-9, 2008.

[90] C. Wang, M. Liu, Q. Wen, and N. Li, "Clinical observation of preventing and treating constipation by electroacupuncture intervention in ischemic stroke patients at acute stage," Zhongguo Zhen Jiu, vol. 35, no. 5, pp. 430-434, 2015.

[91] W. B. Fu, Y. Guo, X. K. Chen et al., "Comprehensive therapeutic protocol of electroacupuncture combined with Chinese herbs and rehabilitation training for treatment of cerebral infarction: a multi-center randomized controlled trial," Zhongguo Zhen Jiu, vol. 30, no. 1, pp. 6-9, 2010.

[92] Q. Tang, L. Zhang, T. H. Wei, Y. Wang, J. Bai, and H. T. Li, "Randomized controlled clinical trials for electro-spoonneedles treatment of motor dysfunction in cerebral infarction patients," Zhen Ci Yan Jiu, vol. 34, no. 3, pp. 193-197, 2009.

[93] X. P. Qian, F. Xu, J. L. Song, and J. H. Zhao, "Influence of different frequencies of acupuncture on therapeutic effect in patients with cerebral infarction at convalescence," Zhongguo Zhen Jiu, vol. 29, no. 1, pp. 7-9, 2009.

[94] J. Li, "Forty-five cases of apoplexy treated by electroacupuncture at the points of yin meridians," Journal of Traditional Chinese Medicine, vol. 21, no. 1, pp. 20-22, 2001.

[95] A. M. K. Wong, T.-Y. Su, F.-T. Tang, P.-T. Cheng, and M.-Y. Liaw, "Clinical trial of electrical acupuncture on hemiplegic stroke Patients1," American Journal of Physical Medicine \& Rehabilitation, vol. 78, no. 2, pp. 117-122, 1999.

[96] X. Wang, L. Yang, and Y. Liu, "Clinical observation on treatment of ischemic apoplexy by electroacupuncture plus acupoint-injection," Journal of Traditional Chinese Medicine= Chung I Tsa Chih Ying Wen pan, vol. 27, no. 3, pp. 175-177, 2007.

[97] J. Li, Y. Hu, L. Tong, D. Wang, and D. Zhang, "Effects of electroacupuncture plus intra-carotid drug injection on rheoencephalogram in patients with cerebral infarction," Journal of Traditional Chinese Medicine = Chung I Tsa Chih Ying Wen pan, vol. 24, no. 1, pp. 28-29, 2004.

[98] J. Pei, L. Sun, R. Chen, T. Zhu, Y. Qian, and D. Yuan, “The effect of electro-acupuncture on motor function recovery in patients with acute cerebral infarction: a randomly controlled trial," Journal of Traditional Chinese Medicine = Chung I Tsa Chih Ying Wen pan, vol. 21, no. 4, pp. 270-272, 2001.

[99] C. Wang, Z. Wu, N. Li et al., "Clinical curative effect of electric acupuncture on acute cerebral infarction: a randomized controlled multicenter trial," Journal of Traditional Chinese Medicine, vol. 34, no. 6, pp. 635-640, 2014. 